\title{
Lists that are smaller than their parts: A coding approach to tunable secrecy
}

\author{
Flávio du Pin Calmon ${ }^{\dagger}$, Muriel Médard ${ }^{\dagger}$, Linda M. Zeger ${ }^{\ddagger}$, \\ João Barros*, Mark M. Christiansen**, Ken R. Duffy** \\ ${ }^{\dagger}$ Massachusetts Institute of Technology, Cambridge, MA, \{flavio, medard $\} @$ mit.edu \\ ${ }^{\ddagger}$ MIT Lincoln Laboratory, Lexington, MA, zeger@auroral.biz \\ * Instituto de Telecommunicações, FEUP, Porto, Portugal, jbarros@fe.up.pt \\ ** Hamilton Institute, National University of Ireland, Maynooth, Ireland, \{mark.christiansen, ken.duffy $\} @$ nuim.ie
}

\begin{abstract}
We present a new information-theoretic definition and associated results, based on list decoding in a source coding setting. We begin by presenting list-source codes, which naturally map a key length (entropy) to list size. We then show that such codes can be analyzed in the context of a novel information-theoretic metric, $\epsilon$ symbol secrecy, that encompasses both the one-time pad and traditional rate-based asymptotic metrics, but, like most cryptographic constructs, can be applied in nonasymptotic settings. We derive fundamental bounds for $\epsilon$ symbol secrecy and demonstrate how these bounds can be achieved with MDS codes when the source is uniformly distributed. We discuss applications and implementation issues of our codes.
\end{abstract}

\section{INTRODUCTION}

Classic information-theoretic approaches to secrecy are concerned with unconditionally secure systems, i.e. schemes that manage to hide all the bits of a message from an adversary with unbounded computational resources. It is well known that, for a noiseless setting, unconditional (i.e. perfect) secrecy can only be attained when both communicating parties share a random key with entropy at least as large as the message itself [1]. In other cases, perfect secrecy can sometimes be achieved by exploiting particular characteristics of the considered model, such as when the legitimate communicating party has a less noisy channel than the eavesdropper (wiretap channel) [2].

Alternatively, computationally secure cryptosystems have thrived both from a theoretical and a practical

This work is sponsored by the Department of Defense under Air Force Contract FA8721-05-C-0002. Opinions, interpretations, recommendations, and conclusions are those of the authors and are not necessarily endorsed by the United States Government. Specifically, this work was supported by Information Systems of ASD(R\&E).

$\ddagger$ Currently with Auroral LLC.

** Supported by the Irish Higher Educational Authority (HEA) PRTLI Network Mathematics Grant. perspective. Such systems are based on yet unproven hardness assumptions, but nevertheless have led to cryptographic schemes that are widely adopted (for an overview, see [3]). Currently, computationally secure schemes are used millions of times per day, in applications that range from online banking transactions to digital rights management. However, with the ever increasing amount of data streaming over the Internet and the need to provide secure connections to mobile low powered devices, there is still a constant demand for new and efficient security solutions.

There has been a long exploration of the connection between coding and cryptography [4], and our work is inscribed in this school of thought. From a theoretical perspective, we aim to present a new framework that allows the application of information theoretic-tools to analyze a broader set of secrecy schemes that go beyond the one-time pad and the wiretap model with its variations. Towards this goal, we define a new metric for analyzing security, namely $\epsilon$-symbol secrecy, which quantifies the uncertainty of specific source symbols given an encrypted source sequence. This metric subsumes traditional rate-based information-theoretic measures of secrecy which, unlike usual cryptographic approaches, are generally asymptotic. However, our definition is not asymptotic and, indeed, we provide a construction that achieves fundamental symbol secrecy bounds, based on MDS codes, for finite-length sequence.

In order to construct schemes that achieve symbol secrecy performance bounds, we present the definition of list-source codes, which are codes that compress a source sequence below its entropy rate. Consequently, a list-source code is decoded to a list of possible source sequences instead of a unique source sequence. Fundamental bounds for list-source codes are derived, and explicit constructions that achieve such bounds are 
presented using tools from algebraic coding theory.

We show how list-source codes can be used as an important tool for hiding information with key sizes that are only a fraction of the entropy of the message. Using list-source codes, it becomes possible to argue that the best an adversary can do is to reduce the set of possible messages to an exponentially sized list with certain properties, where the size of the list depends on the length of the key. Since the list has an exponential size, it cannot be resolved in polynomial time, offering a certain level of computational security. We will show how this property can be used to develop hybrid encryption schemes, where only part of the message needs to be securely encrypted.

Our main practical application of interest is secure content caching and distribution. We propose a hybrid encryption scheme based on list-source codes, where a large fraction of the message can be encoded and distributed using a key-independent list-source code. The information necessary to resolve the decoding list, which can be much smaller than the whole message, is then encrypted using a secure method. This scheme allows a significant amount of content to be distributed and cached before dealing with key generation, distribution and management issues.

\section{A. Related work}

Tools from algebraic coding theory have been widely used for constructing secrecy schemes [4]. In addition, the notion of providing security by exploiting the fact that the adversary has incomplete access to information is also central to several secure network coding schemes and wiretap models. Ozarow and Wyner [5] introduced the wiretap channel II, where an adversary can observe a set $k$ of his choice out of $n$ transmitted symbols, and proved that there exists a code that achieves perfect secrecy. A generalized version of this model was investigated by Cai and Yeung in [6], where they introduce the related problem of designing an information-theoretically secure linear network code when an adversary can observe a certain number of edges in the network. Their results were later extended in [7][10]. A more practical approach was presented by Lima et al. in [11]. For a survey on the theory of secure network coding, we refer the reader to [12].

The setting considered in this paper is related to the wiretap channel II in that a fraction of the source symbols is hidden from a possible adversary. Oliveira et al. investigated in [13] a related setting in the context of data storage over untrusted networks that do not collude, introducing a solution based on Vandermonde matrices. The MDS coding scheme introduced in this paper is similar to [13], albeit the framework developed here is more general.

List decoding techniques for channel coding were first introduced by Elias [14] and Wozencraft [15], with subsequent work by Shannon et al. [16], [17] and Forney [18]. Recently, new algorithmic results for list decoding of channel codes were discovered by Gurusuwami and Sudan [19]. We refer the reader to [20] for an excellent survey of list decoding results. List decoding has been considered in the context of source coding in [21]. The approach is related to the one presented here, since we may view a secret key as side information, but [21] do not consider source coding and list decoding together for the purposes of security.

\section{B. Communication and threat model}

A transmitter (Alice) sends to a legitimate receiver (Bob) a sequence of length $n$ produced by a discrete source $X$ with output alphabet $\mathcal{X}$ and probability distribution $p_{X}(\cdot)$. Both Alice and Bob have access to a shared secret key $K$ drawn uniformly and at random from a discrete alphabet $\mathcal{K}$, such that $H(K)<H\left(X^{n}\right)$, and encryption/decryption functions Enc : $\mathcal{X}^{n} \times \mathcal{K} \rightarrow \mathcal{C}$ and Dec : $\mathcal{C} \times \mathcal{K} \rightarrow \mathcal{X}^{n}$, where $\mathcal{C}$ is the set of possible encrypted messages. In addition, Alice communicates with Bob over a noiseless channel. Alice observes the source sequence $X^{n}$, and transmits an encrypted message $C=\operatorname{Enc}\left(X^{n}, K\right)$. Bob then recovers $X^{n}$ by decrypting the message using the key, recovering $\hat{X}^{n}=\operatorname{Dec}(C, K)$. The communication is successful if $\hat{X}^{n}=X^{n}$

We assume a passive but computationally unbounded eavesdropper (Eve) that has access to all transmitted messages from Alice to Bob and knows the functions $\operatorname{Enc}(\cdot)$ and $\operatorname{Dec}(\cdot)$, but does not know the secret key $K$. Eve's goal is to gain as much knowledge as possible about the original source sequence. This is the traditional framework used in cryptography, and no degraded assumption is made beyond the shared secret key.

In the remainder of this paper we investigate two main aspects of this model, described below.

1) Encryption with key entropy smaller than the message entropy: We initially analyze how to perform encryption when the key is smaller than the message. Towards this goal, we present the definition of listsource codes (LSCs), together with fundamental bounds, in section III Furthermore, practical code constructions of LSCs are introduced in section III. We present listsource codes as codes that compress the source sequence below its entropy rate, and in section III describe how LSCs can be used in the considered model. 
2) Security analysis and new security metrics for i.i.d. sources: We analyze the security of schemes based on LSCs in section IV In addition, we introduce a new information-theoretic metric that can be used in scenarios where perfect secrecy cannot be achieved, namely absolute and $\epsilon$-symbol secrecy.

In section $\mathrm{V}$ we discuss the extension of LSCs to Markovian source models, and in section VI we present applications and practical considerations of the proposed secrecy scheme. Finally, section VII presents our concluding remarks.

\section{LIST DECODING AND SOURCE CODING: FUNDAMENTAL LIMITS}

In this section we present the definition of list-source codes and derive fundamental bounds. Consider a discrete memoryless source $X$ with output alphabet $\mathcal{X}$ and probability distribution $p_{X}(\cdot)$.

Definition 1. A $\left(2^{n R},|\mathcal{X}|^{n L}, n\right)$-list-source code for a discrete memoryless source $X$ consists of an encoding function $f_{n}: \mathcal{X}^{n} \rightarrow\left\{1, \ldots, 2^{n R}\right\}$ and a list-decoding function $g_{n}:\left\{1, \ldots, 2^{n R}\right\} \rightarrow \mathcal{P}\left(\mathcal{X}^{n}\right) \backslash \varnothing$, where $\mathcal{P}\left(\mathcal{X}^{n}\right)$ is the power set of $\mathcal{X}^{n}$ and $|g(w)|=|\mathcal{X}|^{n L} \forall w \in$ $\left\{1, \ldots, 2^{n R}\right\}$.

Note that $0 \leq L \leq 1$. From an operational point of view, $L$ is a parameter that determines the size of the decoded list. For example, $L=0$ corresponds to traditional lossless compression, i.e., each source sequence is decoded to a unique sequence. Furthermore, $L=1$ represents the trivial case when the decoded list corresponds to $\mathcal{X}^{n}$.

For a list-source code, an error is declared when a string generated by a source is not contained in the corresponding decoded list. The average error probability is given by

$$
e_{L}\left(f_{n}, g_{n}\right)=\operatorname{Pr}\left(X^{n} \notin g_{n}\left(f_{n}\left(X^{n}\right)\right)\right) .
$$

Definition 2. For a given discrete memoryless source $X$, the rate list size pair $(R, L)$ is said to be achievable if for every $\delta>0,0<\epsilon<1$ and sufficiently large $n$ there exists a sequence of $\left(2^{n R_{n}},|\mathcal{X}|^{n L_{n}}, n\right)$-list-source codes $\left(f_{n}, g_{n}\right)$ such that $R_{n}<R+\delta,\left|L_{n}-L\right|<\delta$ and $e_{L_{n}}\left(f_{n}, g_{n}\right) \leq \epsilon$. The rate list region is the closure of all rate list pairs $(R, L)$.

Definition 3. The rate list function $R(L)$ is the infimum of all rates $R$ such that $(R, L)$ is in the rate list region for a given normalized list size $0 \leq L \leq 1$.

Proposition 1. For any discrete memoryless source X, the rate list function is bounded below by

$$
R(L) \geq H(X)-L \log |\mathcal{X}|
$$

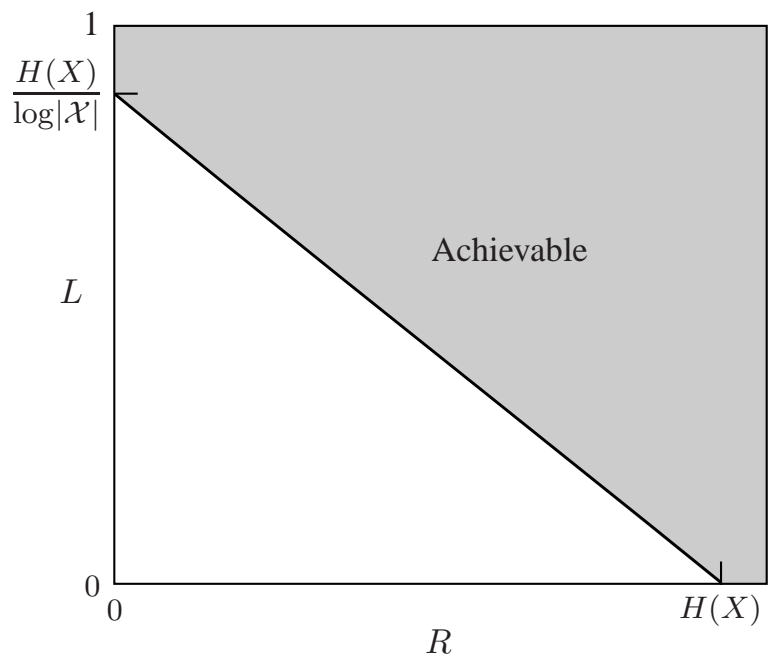

Fig. 1. Rate list region for normalized list size $L$ and code rate $R$.

Proof: Let $\delta>0$ be given and $\left(f_{n}, g_{n}\right)$ be a sequence of codes with (normalized) list size $L_{n}$ such that $L_{n} \rightarrow L$ and for any $0<\epsilon<1$ and $n$ sufficiently large $0 \leq e_{L}\left(f_{n}, g_{n}\right) \leq \epsilon$. Then

$$
\begin{aligned}
\operatorname{Pr}\left[X^{n} \in \bigcup_{w \in \mathcal{W}^{n}} g_{n}(w)\right] & \geq \operatorname{Pr}\left[X^{n} \in g_{n}\left(f_{n}\left(X^{n}\right)\right)\right] \\
& \geq 1-\epsilon
\end{aligned}
$$

where $\mathcal{W}^{n}=\left\{1, \ldots, 2^{n R_{n}}\right\}$ and $R_{n}$ is the rate of the code $\left(f_{n}, g_{n}\right)$. Using [22, Lemma 2.14]:

$$
\begin{aligned}
\frac{1}{n} \log \left(\sum_{w \in \mathcal{W}^{n}}\left|g_{n}(w)\right|\right) & =\frac{1}{n} \log \left(2^{n R_{n}}|\mathcal{X}|^{n L_{n}}\right) \\
& =R_{n}+L_{n} \log |\mathcal{X}| \\
& \geq \frac{1}{n} \log \left|\bigcup_{w \in \mathcal{W}^{n}} g_{n}(w)\right| \\
& \geq H(X)-\delta
\end{aligned}
$$

if $n \geq n_{0}(\delta, \epsilon,|\mathcal{X}|)$. Since this holds for any $\delta>0$, it follows that $R(L) \geq H(X)-L \log |\mathcal{X}|$ for all $n$ sufficiently large.

Remark 1. Achievability of the bound (2) will be shown through an explicit design using linear codes in the next section, so the inequality can be proved to be an equality.

\section{CODE DESIGN}

\section{A. Trivial approach}

Assume that the source $X$ is uniformly distributed in $\mathbb{F}_{q}$, i.e., $\operatorname{Pr}(X=x)=1 / q \forall x \in \mathbb{F}_{q}$. In this case 
$R(L)=(1-L) \log q$. A trivial scheme for achieving the list-source boundary is the following. Consider a source sequence $X^{n}=\left(X^{p}, X^{s}\right)$, where $X^{p}$ denotes the first $p=n-\lfloor L n\rfloor$ symbols of $X^{n}$ and $X^{s}$ denotes the last $s=\lfloor L n\rfloor$ symbols. Encoding is done by discarding $X^{s}$, and mapping the prefix $X^{p}$ to a binary codeword $Y^{n R}$ of length $n R=\lceil n-\lfloor L n\rfloor \log q\rceil$ bits.

For decoding, the codeword $Y^{n R}$ is mapped to $X^{p}$, and the scheme outputs a list of size $q^{s}$ composed by $X^{p}$ concatenated with all possible combinations of suffixes of length $s$. Clearly, for $n$ sufficiently large, $R \approx(1-L) \log q$, and we achieve the optimal list-source size tradeoff.

The previous scheme is completely inadequate for security purposes. An adversary that observes the binary codeword $Y^{n R}$ can uniquely identify the first $p$ symbols of the source message, and the uncertainty is concentrated over the last $s$ symbols. Ideally, assuming that all source symbols are of equal importance, we should spread the uncertainty over all symbols of the message. More precisely, given the encoding $f\left(X^{n}\right)$, a "good" security scheme would provide $I\left(X_{i} ; f\left(X^{n}\right)\right) \leq \epsilon \ll \log q$ for $1 \leq i \leq n$. Of course, we can naturally extend this notion for groups of symbols or functions over input symbols 1 . This idea will be captured in the definition of symbol secrecy, introduced in section IV.

\section{B. A construction based on linear codes}

Let $X$ be an i.i.d. source with $X \in \mathcal{X}$ with entropy $H(X)$, and $\mathcal{S}_{n}$ a source code with encoder $s_{n}: \mathcal{X}^{n} \rightarrow \mathbb{F}_{q}^{m_{n}}$ and decoder $r_{n}: \mathbb{F}_{q}^{m_{n}} \rightarrow \mathcal{X}^{n}$. Furthermore, let $\mathcal{C}$ be a $\left(m_{n}, k_{n}, d\right)$ linear code over $\mathbb{F}_{q}$ with an $\left(m_{n}-k_{n}\right) \times m_{n}$ parity check matrix $\mathbf{H}_{n}$ (i.e. $\mathbf{c} \in \mathcal{C} \Leftrightarrow \mathbf{H}_{n} \mathbf{c}=0$ ). Consider the following scheme, where $k_{n}=n L_{n} \log |\mathcal{X}| / \log q$ for $0 \leq L_{n} \leq 1$ and $L_{n} \rightarrow L$ as $n \rightarrow \infty$. To simplify notation, we assume without loss of generality that $k_{n}$ is an integer.

Scheme 1. Encoding: Let $X^{n}$ be the sequence generated by the source. Compute the syndrome $S^{m_{n}-k_{n}}=$ $\mathbf{H}_{n} s_{n}\left(X^{n}\right)$ and map each syndrome to a distinct sequence of $n R=\left\lceil\left(m_{n}-k_{n}\right) \log q\right\rceil$ bits, denoted by $Y^{n R}$

Decoding: Map the binary codeword $Y^{n R}$ to the corresponding syndrome $S^{m_{n}-k_{n}}$. Output $r_{n}\left(x^{m_{n}}\right)$ for each $x^{m_{n}}$ in the coset of $\mathbf{H}_{n}$ corresponding to $S^{m_{n}-k_{n}}$.

Proposition 2. If $\mathcal{S}_{n}$ is asymptotically optimal for source $X$, i.e. $m_{n} / n \rightarrow H(X) / \log q$, scheme 1 achieves the optimal list-source tradeoff point $R(L)$ for an i.i.d. source, where $R(\cdot)$ is the rate list function.

\footnotetext{
${ }^{1}$ This idea is tightly related to the concept of hard core predicates and semantic security in cryptography.
}

Proof: Since the size of each coset corresponding to a syndrome a $S^{m_{n}-k_{n}}$ is exactly $q^{k_{n}}$, the normalized list size is $L_{n}=\left(k_{n} \log q\right) /(n \log |\mathcal{X}|) \rightarrow L$. Denoting $m_{n} / n=H(X) / \log q+\delta_{n}$, where $\delta_{n} \rightarrow 0$, it follows that is $R=\left\lceil\left(m_{n}-k_{n}\right) \log q\right\rceil / n=\left\lceil\left(H(X)+\delta_{n} \log q\right) n-\right.$ $\left.L_{n} n \log |\mathcal{X}|\right\rceil / n$, which is arbitrarily close to the rate in (2) for sufficiently large $n$.

The source coding scheme used in the proof of Proposition 2 can be any asymptotically optimal scheme. Note that if the source $X$ is uniform, and assuming without loss of generality that $L_{n}=L$ and that $L n$ is an integer, any message in the coset of $\mathcal{C}$ determined by $S^{(1-L) n}$ is equally likely. Hence, $H\left(X^{n} \mid S^{(1-L) n}\right)=q^{L n}$. Scheme 1 provides a systematic way of hiding information, and we can take advantage of the properties of the underlying linear code to make precise assertions regarding the "information leakage" of the scheme.

With the syndrome in hand, how can we recover the rest of the message? One possible approach is to find a $k \times n$ matrix $\mathbf{D}$ that has full rank such that the rows of $\mathbf{D}$ and $\mathbf{H}$ form a basis of $\mathbb{F}_{q}^{n}$. Such a matrix can be easily found, for example, using the Gram-Schmidt process with the rows of $\mathbf{H}$ as a starting point. Then we simply calculate $T^{L n}=\mathbf{D} X^{n}$ and forward $T^{L n}$ to the receiver. The receiver can then invert the system

$$
\left(\begin{array}{l}
\mathbf{H} \\
\mathbf{D}
\end{array}\right) X^{n}=\left(\begin{array}{c}
S^{(1-L) n} \\
T^{L n}
\end{array}\right),
$$

and recover the original sequence $X^{n}$. This property allows list-source codes to be deployed in practice using well known linear code constructions, such as ReedSolomon or LDPC.

Remark 2. This approach is valid for general linear spaces, and holds for any pair of full rank matrices $\mathbf{H}$ and $\mathrm{D}$ with dimensions $(n-k) \times n$ and $k \times n$, respectively, such that $\operatorname{rank}\left(\left[\mathbf{H}^{T} \mathbf{D}^{T}\right]^{T}\right)=n$. However, here we adopt the nomenclature of linear codes since we make use of known code constructions to design secrecy schemes in the following sections.

\section{A secure communication scheme based on list-source codes}

In this section we present a general description of a two-phase secure communication scheme for the model introduced in section $\amalg$-B presented in terms of the listsource code constructions derived using linear codes. Note that this scheme can be easily extended to any list-source code by using the corresponding encoding/decoding functions instead of multiplication by parity check matrices.

We assume that Alice and Bob have access to a encryption/decryption scheme $\left(\mathrm{Enc}^{\prime}, \mathrm{Dec}^{\prime}\right)$ that is used 
with the shared secret key $K$ and is sufficiently secure against the adversary. This scheme can be, for example, a one-time pad. The encryption/decryption procedure is performed as follows, and will be used as components of the overall encryption scheme (Enc, Dec) described below.

Scheme 2. Input: The source encoded sequence $X^{n} \in$ $\mathbb{F}_{q}^{n}$, parity check matrix $\mathbf{H}$ of a linear code in $\mathbb{F}_{q}^{n}$, a fullrank $k \times n$ matrix $\mathbf{D}$ such that $\operatorname{rank}\left(\left[\mathbf{H}^{T} \mathbf{D}^{T}\right]\right)=n$, and encryption/decryption functions $\left(\mathrm{Enc}^{\prime}, \mathrm{Dec}^{\prime}\right)$.

\section{Encryption (Enc):}

Phase I (pre-caching): Alice generates $S^{n-k}=\mathbf{H} X^{n}$ and sends to Bob.

Phase II (send encrypted data): Alice generates $E^{k}=$ $\operatorname{Enc}^{\prime}\left(\mathbf{D} X^{n}, K\right)$ and sends to Bob.

Decryption (Dec): Bob calculate $\mathbf{D} X^{n}=\operatorname{Dec}^{\prime}\left(E^{k}\right)$ and recover $X^{n}$ from $S^{n-k}$ and $\mathbf{D} X^{n}$.

Assuming that $\left(\mathrm{Enc}^{\prime}, \mathrm{Dec}^{\prime}\right)$ is secure, the security of scheme 2 reduces to the security of the underlying listsource code (i.e. scheme 11). In practice, the encryption/decryption functions (Enc', Dec') may depend on a secret or public/private key, as long as it provide sufficient security for the desired application. In addition, assuming that the source sequence is uniform and i.i.d. in $F_{q}^{n}$, we can use MDS codes to make strong security guarantees, as described in the next section. In this case, an adversary that observes $S^{n-k}$ cannot infer any information about any set of $k$ symbols of the original message.

Note that this scheme has a tunable level of secrecy: The amount of data sent in phase I and phase II can be appropriately selected to match the properties of the encryption scheme available, the size of the key length, and the desired level of secrecy. Furthermore, when the encryption procedure has a higher computational cost than the list-source encoding/decoding operations, listsource codes can be used to reduce the total number of operations required by allowing encryption of a smaller portion of the message (phase II).

\section{NEW METRICS FOR SECURITY ANALYSIS}

We introduce a new information-theoretic metric for security called $\epsilon$-symbol secrecy. This metric can be used to characterize the properties of security schemes that do not provide absolute secrecy (such as in scheme 2). Given a source sequence $X^{n}$ and its corresponding encryption $Y, \epsilon$-symbol secrecy is the largest fraction $t / n$ such that at most $\epsilon$ bits can be inferred from any $t$-symbol subsequence of $X^{n}$. We derive a fundamental bound for $\epsilon$-symbol secrecy, and show that it can be achieved using MDS codes for $\epsilon=0$ and uniform i.i.d. sources. Before presenting the definition, we make a few comments on notation and briefly review the threat model.

\section{A. Notation}

Let $\mathcal{C}_{n}$ be a sequence of codes for a discrete memoryless source $X$ with probability distribution $p(x)$ that achieves a rate list pair $(R, L)$. Furthermore, let $Y^{n R_{n}}$ be the corresponding codeword $f_{n}\left(X^{n}\right)$ created by $\mathcal{C}_{n}$. Denote by $\mathcal{I}_{n}(t)$ the set of all subsets of $\{1, \ldots, n\}$ of size $t$, i.e. $\mathcal{J} \in \mathcal{I}_{n}(t) \Leftrightarrow \mathcal{J} \subseteq\{1, \ldots, n\}$ and $|\mathcal{J}|=t$. In addition, we denote by $X^{(\mathcal{J})}$ the set of symbols of $X^{n}$ indexed by the elements in the set $\mathcal{J} \subseteq\{1, \ldots, n\}$.

As discussed in section I-B, we assume a passive but computationally unbounded adversary that only has access to the list-source encoded message $f_{n}\left(X^{n}\right)=$ $Y^{n R_{n}}$. Based on the observation of $Y^{n R_{n}}$, the adversary will attempt to determine what is the original message. In addition, we assume that the source statistics and the list-source code used are universally known, i.e. an adversary has access to the distribution $p_{X^{n}}\left(X^{n}\right)$ of the symbol sequences produced by the source and the sequence of codes $\mathcal{C}_{n}$. We use the standard informationtheoretic approach of measuring the amount of information that an adversary can gain of a specific sequence of source symbols $X^{(\mathcal{J})}$ by observing $Y^{n R_{n}}$ as the mutual information $I\left(X^{(\mathcal{J})} ; Y^{n R_{n}}\right)$.

\section{B. Symbol Secrecy}

The following definition introduces two security metrics, namely absolute symbol secrecy and $\epsilon$-symbol secrecy.

Definition 4. We define $\mu_{0}\left(\mathcal{C}_{n}\right)$ as the absolute symbol secrecy of a code $\mathcal{C}_{n}$ as

$\mu_{0}\left(\mathcal{C}_{n}\right)=\max \left\{\frac{t}{n}: I\left(X^{(\mathcal{J})} ; Y^{n R_{n}}\right)=0, \forall \mathcal{J} \in \mathcal{I}_{n}(t)\right\}$.

The absolute symbol secrecy $\mu_{0}$ of a sequence of codes $\mathcal{C}_{n}$ is:

$$
\mu_{0}=\liminf _{n \rightarrow \infty} \mu_{0}\left(\mathcal{C}_{n}\right) .
$$

Furthermore, we define the $\epsilon$-symbol secrecy $\mu_{\epsilon}$ of a code $\mathcal{C}_{n}$ as

$\mu_{\epsilon}\left(\mathcal{C}_{n}\right)=\max \left\{\frac{t}{n}: \frac{1}{t} I\left(X^{(\mathcal{J})} ; Y^{n R_{n}}\right) \leq \epsilon \forall \mathcal{J} \in \mathcal{I}_{n}(t)\right\}$,

and the $\epsilon$-symbol secrecy of a sequence of codes $\mathcal{C}_{n}$ as

$$
\mu_{\epsilon}=\liminf _{n \rightarrow \infty} \mu_{\epsilon}\left(\mathcal{C}_{n}\right),
$$

where $\epsilon<H(X)$. 
Proposition 3. Let $\mathcal{C}_{n}$ be a sequence of list-source codes that achieves a rate-list pair $(R, L)$ and an $\epsilon$-symbol secrecy of $\mu_{\epsilon}$. Then $0 \leq \mu_{\epsilon} \leq \min \left\{\frac{L \log |\mathcal{X}|}{H(X)-\epsilon}, 1\right\}$.

Proof: We denote $\mu_{\epsilon}\left(\mathcal{C}_{n}\right)=\mu_{\epsilon, n}$. Note that

$$
\begin{aligned}
I\left(X^{(\mathcal{J})} ; Y^{n R_{n}}\right) & =H\left(X^{(\mathcal{J})}\right)-H\left(X^{(\mathcal{J})} \mid Y^{n R_{n}}\right) \\
& =n \mu_{\epsilon, n} H(X)-H\left(X^{(\mathcal{J})} \mid Y^{n R_{n}}\right) \\
& \leq n \mu_{\epsilon, n} \epsilon .
\end{aligned}
$$

Therefore

$$
\begin{aligned}
\mu_{\epsilon, n}(H(X)-\epsilon) & \leq \frac{1}{n} H\left(X^{(\mathcal{J})} \mid Y^{n R_{n}}\right) \\
& \leq L_{n} \log |\mathcal{X}| .
\end{aligned}
$$

The result follows by taking $n \rightarrow \infty$.

The previous result bounds the amount of information an adversary gains about particular source symbols by observing a list-source encoded message. In particular, for $\epsilon=0$, we find a meaningful bound on what is the largest fraction of input symbols that is perfectly hidden. A simple upper-bound for the maximum average amount of information that an adversary can gain from a message encoded with any source code $\mathcal{C}_{n}$ with symbol secrecy $u_{\epsilon, n}$ is given below.

Proposition 4. For any code $\mathcal{C}_{n}$ for a discrete memoryless source $X$ and any $\epsilon$ such that $0 \leq \epsilon \leq H(X)$, we have

$$
\frac{1}{n} I\left(X^{n} ; Y^{n R_{n}}\right) \leq H(X)-\mu_{\epsilon, n}(H(X)-\epsilon),
$$

where $\mu_{\epsilon, n}=\mu_{\epsilon}\left(\mathcal{C}_{n}\right)$.

Proof: Let $\mu_{\epsilon, n}=t / n, \mathcal{J} \in \mathcal{I}_{n}(t)$ and $\overline{\mathcal{J}}=$ $\{1, \ldots, n\} \backslash \mathcal{J}$. Then

$$
\begin{aligned}
\frac{1}{n} I\left(X^{n} ; Y^{n R_{n}}\right) & \leq \frac{t}{n}\left(\epsilon+\frac{1}{t} I\left(X^{(\overline{\mathcal{J}})} ; Y^{n R_{n}} \mid X^{(\mathcal{J})}\right)\right) \\
& \leq \mu_{\epsilon, n} \epsilon+\frac{(n-t)}{n} H(X) \\
& =H(X)-\mu_{\epsilon, n}(H(X)-\epsilon) .
\end{aligned}
$$

The next proposition relates the rate-list function with $\epsilon$-symbol secrecy through the upper bound in proposition 3

Proposition 5. If a sequence of list-source codes $\mathcal{C}_{n}$ achieves a point $\left(R^{\prime}, L\right)$ with $\mu_{\epsilon}=\frac{L \log |\mathcal{X}|}{H(X)-\epsilon}$ for some $\epsilon$, where $R^{\prime}=\lim _{n \rightarrow \infty} \frac{1}{n} H\left(Y^{n R_{n}}\right)$, then $R^{\prime}=R(L)$.

Proof: Assume that $\mathcal{C}_{n}$ satisfies the conditions in the proposition and $\delta>0$ is given. Then for $n$ sufficiently large, we have from 111:

$$
\begin{aligned}
\frac{1}{n} H\left(Y^{n R_{n}}\right) & =\frac{1}{n} I\left(X^{n} ; Y^{n R_{n}}\right) \\
& \leq H(X)-\mu_{\epsilon}(H(X)-\epsilon)+\delta \\
& =H(X)-L \log |\mathcal{X}|+\delta .
\end{aligned}
$$

Since this holds for any $\delta$, then $R^{\prime} \leq H(X)-L \log |\mathcal{X}|$. However, from proposition $1, R^{\prime} \geq H(X)-L \log |\mathcal{X}|$, and the result follows.

\section{A scheme based on MDS codes}

We now prove that for a uniform i.i.d. source $X$ in $\mathbb{F}_{q}$, using scheme 1 with an MDS parity check matrix $\mathbf{H}$ achieves $\mu_{0}$. Since the source is uniform and i.i.d., no source coding is used.

Proposition 6. If $\mathbf{H}$ is the parity check matrix of an $(n, k, d) M D S$ and the source $X^{n}$ is uniform and i.i.d., then Scheme 1 achieves the upper bound $\mu_{0}=L$, where $L=k / n$.

Proof: Let $\mathbf{H}$ be the parity check matrix of a $(n, k, n-k+1)$ MDS code $\mathcal{C}$ over $\mathbb{F}_{q}$, and let $\mathbf{x} \in \mathcal{C}$. Fix a set $\mathcal{J} \in \mathcal{I}_{n}(k)$ of $k$ positions of $\mathbf{x}$, denoted $\mathbf{x}^{(\mathcal{J})}$. Since the minimum distance of $\mathcal{C}$ is $n-k+1$, for any other codeword in $\mathbf{z} \in \mathcal{C}$ we have $\mathbf{z}^{(\mathcal{J})} \neq \mathbf{x}^{(\mathcal{J})}$. Denoting by $\mathcal{C}^{(\mathcal{J})}=\left\{x^{(\mathcal{J})} \in \mathbb{F}_{q}^{k}: x \in \mathcal{C}\right\}$, then $\left|\mathcal{C}^{(\mathcal{J})}\right|=|\mathcal{C}|=q^{k}$. Therefore, $\mathcal{C}^{(\mathcal{J})}$ contains all possible combinations of $k$ symbols. Since this property also holds for any coset of $\mathbf{H}$, the result follows.

\section{LIST-SOURCE CODES FOR GENERAL SOURCE MODELS}

The previous results hold for i.i.d. source models. However, for more general sources the analysis becomes significantly more convoluted, since multiple list-source encoded messages can reveal information about each other. Considering that encryption is performed over multiple blocks of source symbols, the list size will not necessarily grow if these block are correlated.

In general, given an output $\mathbf{X}=X_{1}, \ldots, X_{n}$ of $n$ correlated source symbols, and using scheme 1 , what is observed by an eavesdropper is the coset valued sequence of random elements $\left\{H\left(s_{n}(\mathbf{X})\right)\right\}, H$ being the parity check matrix. Since $\mathbf{X}$ is a correlated source of symbols, there is no a priori reason to expect that the coset valued process will not be correlated. For example if $\mathbf{X}$ forms a Markov chain, then the coset valued process is a function of a Markov chain; although it will not, in general, form a Markov chain itself, it will still have correlations. These correlations could effectively reduce the list size that an eavesdropper must search and, 
consequently, reduce the effectiveness of the scheme. Reducing or eliminating correlations in the coset valued process would counteract the impact of this vulnerability.

Different approaches can be taken to resolve this issue. In general, the key to reducing the effect of the correlation between codewords is to encode larger block lengths. More precisely, let $\mathbf{X}_{1}, \mathbf{X}_{2}, \ldots, \mathbf{X}_{N}$ be $N$ blocks of symbols produced by a Markov source, such that $\mathbf{X}_{i} \in \mathcal{X}^{n}$ and $p\left(\mathbf{X}_{1}, \ldots, \mathbf{X}_{N}\right)=$ $p\left(\mathbf{X}_{1}\right) p\left(\mathbf{X}_{2} \mid \mathbf{X}_{1}\right) \ldots p\left(\mathbf{X}_{N} \mid \mathbf{X}_{N-1}\right)$. Instead of encoding each block individually, the transmitter can compute $\mathbf{Y}^{n N R}=f\left(\mathbf{X}_{1}, \ldots, \mathbf{X}_{N}\right)$.

The previous approach has the disadvantage of requiring long block lengths and possibly high implementation complexity. We note, however, that the encoding procedure over multiple blocks does not necessarily have to be performed independently. For example, one possible approach for overcoming edge-effect correlations between codewords is to define $\mathbf{Y}_{1}=f\left(\mathbf{X}_{1}, \mathbf{X}_{2}\right), \mathbf{Y}_{2}=$ $f\left(\mathbf{X}_{2}, \mathbf{X}_{3}\right), \ldots$, and so forth. This approach reduces the edge effects of correlation between codewords, in particular when the individual sequences $\mathbf{X}_{i}$ are already significantly long.

We note that, when probabilistic encryption [3] is required over multiple blocks, the source encoded symbols in scheme 1 can be combined with the output of a pseudorandom number generator (PRG) before being multiplied by the parity check matrix. This would provide the necessary randomization of the output. The initial seed of the PRG can then be transmitted to the legitimate receiver in phase II of scheme 2.

\section{Applications AND PRACTICAL CONSIDERATIONS}

The protocol outline presented in scheme 2 is useful in different practical scenarios, which are discussed in the following sections. Most of the advantages of the suggested scheme stem from the fact that list-source codes are key-independent, allowing content to be distributed when a key distribution infrastructure is not yet established, and providing an additional level of security if keys are compromised before phase II in scheme 2 .

\section{A. Content pre-caching}

As hinted earlier, list-source codes provide a secure mechanism for content pre-caching when a key infrastructure has not yet been established. A large fraction of the data can be list-source coded and securely transmitted before the termination of the key distribution protocol. This is particularly significant in large networks with hundreds of mobile nodes, where key management protocols can require a significant amount of time to complete [23]. Scheme 2 circumvents the communication delays incurred by key compromise detection, revocation and redistribution by allowing data to be efficiently distributed concurrently with the key distribution protocol, while maintaining a level of security determined by the underlying list-source code.

\section{B. Application to key distribution protocols}

List-source codes can also provide additional robustness to key compromise. If the secret key is compromised before phase II of scheme 2, the data will still be as secure as the underlying list-source code. Even if a (computationally unbounded) adversary has perfect knowledge of the key, until the last part of the data is transmitted the best he can do is reduce the number of possible inputs to an exponentially large list. In contrast, if a stream cipher based on a pseudo-random number generator were used and the initial seed was leaked to an adversary, all the data transmitted up to the point where the compromise was detected would be vulnerable. The use of list-source codes provide an additional, information-theoretic level of security to the data up to the point where the last fraction of the message is transmitted. This also allows decisions as to which receivers will be allowed to decrypt the data can be delayed until the very end of the transmission, providing more time for detection of unauthorized receivers and allowing a larger flexibility in key distribution.

In addition, if the level of security provided by the listsource code is considered sufficient and the key is compromised before phase II, the key can be redistributed without the need of retransmitting the entire data. As soon as the keys are reestablished, the transmitter simply encrypts the remaining part of the data in phase II with the new key.

\section{Additional layer of securi ty}

We also highlight that list-source codes can be used to provide an additional layer of security to the underlying encryption scheme. The message can be list-source coded after encryption and transmitted in two phases, as in scheme 2. As argued in the previous point, this provides additional robustness against key compromise, in particular when a compromised key can reveal a large amount of information about an incomplete message (e.g. stream ciphers). Consequently, list-source codes are a simple, practical way of augmenting the security of current encryption schemes.

One example application is to combine list-source codes with stream ciphers, as noted in section V. The source-coded message can be initially encrypted using a pseudorandom number generator initialized with a 
randomly selected seed, and then list-source coded. The initial random seed would be part of the encrypted message sent in the final transmission phase. This setup has the advantage of augmenting the security of the underlying stream cipher, and provides randomization to the list-source coded message. In particular, if the LSC is based on MDS codes and assuming that the distribution of the plaintext is nearly uniform, strong informationtheoretic symbol secrecy guarantees can be made about the transmitted data, as discussed in section IV] Even if the underlying PRG is compromised, the message would still be secure.

\section{Adjustable level of secrecy}

List-source codes provide a tunable level of secrecy, i.e. the amount of security provided by the scheme can be adjusted according to the application of interest. This can be done by appropriately selecting the size of the list $(L)$ of the underlying code, which determines the amount of uncertainty an adversary will have regarding the input message. In the proposed implementation using linear codes, this corresponds to choosing the size of the parity check matrix $\mathbf{H}$, or, analogously, the parameters of the underlying error-correcting code. In terms of scheme 2 a larger (respectively smaller) value of $L$ will lead to a smaller (larger) list-source coded message in phase I and a larger (smaller) encryption burden in phase II.

\section{CONCLUSIONS}

In this paper we introduced the concept of list-source codes, which are codes that compress a source below its entropy rate. We derived fundamental bounds for the rate list region, and provided code constructions that achieve these bounds. List-source codes are a useful tool to understand how to perform encryption when the (random) key length is smaller that the message entropy. In a nutshell, when the key is small, we can reduce an adversary's uncertainty to a near-uniformly distributed list of possible source sequences with an exponential (in terms of the key length) number of elements by using list-source codes. We also demonstrated how list-source codes can be implemented using standard linear codes.

Furthermore, a new information-theoretic metric of secrecy was presented, namely $\epsilon$-symbol secrecy, which characterizes the amount of information leaked about specific symbols of the source given an encoded version of the message. We derived fundamental bounds for $\epsilon$ symbol secrecy, and showed how these bounds can be achieved using MDS codes when the source is uniformly distributed. Finally, we discussed how list-source codes can be applied to practical encryption schemes.

\section{REFERENCES}

[1] C. E. Shannon, "Communication theory of secrecy systems," Bell System Technical Journal, vol. 28, no. 4, pp. 656-715, 1949.

[2] Y. Liang, H. V. Poor, and S. Shamai (Shitz), "Information theoretic security," Found. Trends Commun. Inf. Theory, vol. 5, pp. 355-580, Apr. 2009.

[3] J. Katz and Y. Lindell, Introduction to Modern Cryptography: Principles and Protocols, 1st ed. Chapman and Hall/CRC, Aug. 2007.

[4] R. E. Blahut, D. J. Costello, U. Maurer, and T. Mittelholzer, Eds., Communications and Cryptography: Two Sides of One Tapestry, 1st ed. Springer, Jun. 1994.

[5] L. Ozarow and A. Wyner, "Wire-tap channel II," in Advances in Cryptology, 1985, pp. 33-50.

[6] N. Cai and R. Yeung, "Secure network coding," in IEEE International Symposium on Information Theory, 2002.

[7] J. Feldman, T. Malkin, C. Stein, and R. A. Servedio, "On the capacity of secure network coding," in Proc. 42nd Annual Allerton Conference on Communication, Control, and Computing, 2004.

[8] A. Mills, B. Smith, T. Clancy, E. Soljanin, and S. Vishwanath, "On secure communication over wireless erasure networks," in IEEE International Symposium on Information Theory, Jul. 2008, pp. $161-165$.

[9] S. El Rouayheb, E. Soljanin, and A. Sprintson, "Secure network coding for wiretap networks of type II," IEEE Trans. Inf. Theory, vol. 58, no. 3, pp. 1361 -1371, Mar. 2012.

[10] D. Silva and F. Kschischang, "Universal secure network coding via Rank-Metric codes," IEEE Trans. Inf. Theory, vol. 57, no. 2, pp. $1124-1135$, Feb. 2011.

[11] L. Lima, M. Medard, and J. Barros, "Random linear network coding: A free cipher?" in IEEE International Symposium on Information Theory, Jun. 2007, pp. $546-550$.

[12] N. Cai and T. Chan, "Theory of secure network coding," IEEE Proc., vol. 99, no. 3, pp. 421 -437, Mar. 2011.

[13] P. Oliveira, L. Lima, T. Vinhoza, J. Barros, and M. Medard, "Trusted storage over untrusted networks," in IEEE Global Telecommunications Conference, Dec. 2010, pp. 1 -5.

[14] P. Elias, "List decoding for noisy channels," Research Laboratory of Electronics, MIT, Technical Report 335, September 1957.

[15] J. M. Wozencraft, "List decoding," Research Laboratory of Electronics, MIT, Progress Report 48, 1958.

[16] C. Shannon, R. Gallager, and E. Berlekamp, "Lower bounds to error probability for coding on discrete memoryless channels. I," Information and Control, vol. 10, no. 1, pp. 65-103, Jan. 1967.

[17] - , "Lower bounds to error probability for coding on discrete memoryless channels. II," Information and Control, vol. 10, no. 5, pp. 522-552, May 1967.

[18] G. Forney, "Exponential error bounds for erasure, list, and decision feedback schemes," IEEE Trans. Inf. Theory, vol. 14, no. 2, pp. 206- 220, Mar. 1968.

[19] V. Guruswami, "List decoding of error-correcting codes," Thesis, MIT, Cambridge, MA, 2001.

[20] — " "List decoding of binary Codes-A brief survey of some recent results," in Coding and Cryptology, ser. Lecture Notes in Computer Science. Springer Berlin / Heidelberg, 2009, vol. 5557, pp. 97-106.

[21] M. Ali and M. Kuijper, "Source coding with side information using list decoding," in 2010 IEEE International Symposium on Information Theory Proceedings (ISIT). IEEE, Jun. 2010, pp. 91-95.

[22] I. Csiszár and J. Körner, Information Theory: Coding Theorems for Discrete Memoryless Systems, 2nd ed. Cambridge University Press, Aug. 2011.

[23] L. Eschenauer and V. D. Gligor, "A key-management scheme for distributed sensor networks," in Proceedings of the 9th ACM Conference on Computer and Communications Security, ser. CCS '02. New York, NY, USA: ACM, 2002, pp. 41-47. 